\title{
Optimal Group Sequential Designs and Related (In Clinical Trials)
}

Yanning Liu ${ }^{*}$

Biostatistics and Programming, Janssen Research \& Development, USA

“Corresponding author: Yanning Liu, Biostatistics and Programming, Janssen Research \& Development, LLC, Titusville, NJ, USA, Tel: +86 13636442919; E-mail: YLiu@its.jnj.com

Rec date: May 29, 2016; Acc date: May 30, 2016; Pub date: June 06, 2016

Copyright: (c) 2016 Liu Y. This is an open-access article distributed under the terms of the Creative Commons Attribution License, which permits unrestricted use, distribution, and reproduction in any medium, provided the original author and source are credited.

Citation: Yanning Liu (2016) Optimal Group Sequential Designs and Related (In Clinical Trials). J Biom Biostat 7: 306. doi:10.4172/2155-6180.1000306

\section{Adaptive Designs (in Clinical Trials)}

I have implemented many adaptive design methods using R or SAS, compared them via simulations, have a manuscript under journal review process on a new combination test for designing a two-stage adaptive design. Additionally, I am interested in topics in this area as follows: 1) comments on regulatory guidance documents on adaptive designs ; 2) considerations and optimization of adaptive trial design in clinical development; 3) optimal cost-effective go-no go decisions in clinical development; 4) timing and frequency of interim analyses; 5) approaches for optimal dose selection for adaptive designs; 6) randomization challenges in adaptive design studies; 7) implementing issues; 8) approaches for patient recruitment modeling and simulation.

\section{Survival Analysis}

I've done a couple of papers on issues in clinical trial with survival endpoints: 1) sensitivity analyses for informative censoring in survival data; 2) sample size increase during a survival trial when interim results are promising; 3 ) prediction of the timing of events in clinical trials with survival endpoints; 4) planning a comparative group sequential clinical trial with loss to follow-up and a period of continued observation; 5) planning the duration of a survival group sequential trial with a fixed follow-up time for all subjects.

\section{Statistical Methods in Trials with Sequential Parallel Design for Trials with High Placebo Response}

I've done a few researches in this area as follows: 1) optimal weighted $\mathrm{Z}$ test and linear combination test in extended sequential parallel designs; 2) covariance and variance evaluations of two estimators for drug-placebo difference in a trial with sequential parallel design; 3) misunderstanding of a new approach to drug-placebo difference calculation in short term antidepressant-drug trials; 4) on clinical trials with a high placebo response rate; 5) an unbiased estimator of the two-period treatment effect in doubly randomized delayed-start (DRDS) designs.

\section{Non-Inferiority Designs}

I am also interested in the topics on non-inferiority trials as follows: 1) choice of non-inferiority margin for the mean difference; 2 ) choice of non-inferiority margin for the mean ratio and hazard ratio; 3 ) noninferiority hypotheses with binary endpoints; 4) fixed-margin method and synthesis method; 5)switching between superiority and noninferiority; 6) non-inferiority trials with three treatment groups; 7) regulatory guidance on non-inferiority trials; 8) Intention-to-treat analysis versus per-protocol analysis in non-inferiority trials. 